Digestion 1985;32(suppl. 1):70

\title{
Chairman s Summary
}

\begin{tabular}{|l|l|l|}
\hline A. & Agostinho & Bettarello \\
\hline Based on papers presented at this symposium, it appears that famotidine, a new $\mathrm{H} 2$ receptor
\end{tabular} antagonist, will be effective for the treatment of patients with gastric and duodenal ulcer. It has the advantage of a longer anti-hydrochloric acid activity. In clinical trials comparing famotidine to other $\mathrm{H} 2$ receptor antagonists such as cimetidine or ranitidine, or with other agents such as gefar-nate, the value of the new agent in the treatment of duodenal and gastric ulcer was consistently shown: generally, famotidine was found to be as efficacious as cimetidine and ranitidine and to be much more effective than gefarnate in reversing acid damage. Famotidine has also shown a significantly lower recurrence rate than placebo in patients enrolled in maintenance studies. Furthermore, it seems likely that the longer duration of action of famotidine will be beneficial in the treatment of patients with Zollinger-Ellison syndrome, whose ulcers are directly related to a tumor in the pancreas or in other areas of the gastrointestinal tract.

The safety profile of famotidine is also promising. In a long-term study presented at this symposium, none of the adverse side effects experienced by $5 \%$ of the patients could be directly attributed to drug use. In fact, there have been no documented reports of serious side effects associated with famotidine therapy. The process of ulceration is largely an enigma to physicians and therefore ideal therapy remains elusive. Many questions remain unanswered: why do only $80-85 \%$ of patients with peptic ulcer respond to the currently available treatment? Do ulcers differ etiologically or pathophysiologically or do patients have different genetic make-ups? Are there factors involved in the pathology of the disease that prevent some patients from responding to therapy? Before these questions can be answered types of peptic ulcer must be further categorized so that research can focus on a more homogeneous population. However

for the time being

famotidine appears to be an exciting development for the treatment of ulcers. Further research should focus on long-term therapy and the prevention of ulcer recurrence. With further experience

observation and controlled studies this new agent may prove to be a valuable aid in the management of patients with difficult ulcer disease. 